D. Aksin, S. Gregori, F. Maloberti: "High-Efficiency Power Amplifier for Wireless Sensor Networks"; Proc. of the IEEE International Symposium on Circuits and Systems, ISCAS 2005, Kobe, 23-26 May, Vol. 6, pp. 5898-5901.

(C)20xx IEEE. Personal use of this material is permitted. However, permission to reprint/republish this material for advertising or promotional purposes or for creating new collective works for resale or redistribution to servers or lists, or to reuse any copyrighted component of this work in other works must be obtained from the IEEE. 


\section{High-Efficiency Power Amplifier for Wireless Sensor Networks}

\author{
Devrim Aksin \\ Department of Electrical Engineering \\ University of Texas at Dallas \\ Dallas, Texas, USA \\ devrim.aksin@ieee.org
}

\author{
Stefano Gregori \\ School of Engineering \\ University of Guelph \\ Guelph, Ontario, Canada \\ sgregori@uoguelph.ca
}

\author{
Franco Maloberti \\ Department of Electronics \\ University of Pavia \\ Pavia, Italy \\ franco.maloberti@unipv.it
}

\begin{abstract}
We designed a high-efficiency class-E switchedmode power amplifier for a wireless networked micro-sensors system. In this system, where each sensor operates using a micro-battery, has local processing capability, and contains on the same chip integrated sensing elements and a RF transmitter, most of the power is dissipated by the transmitter. The proposed amplifier achieves $92.4 \%$ maximum drain efficiency and can vary the transmitted power between -4.2 to $-0.2 \mathrm{dBm}$ with almost constant efficiency. This last feature is obtained by controlling the modulation duty cycle and by switching the capacitors' values in the parallel circuit. The possibility of choosing the transmitted power depending on the distance from a base station or other sensors and on the charge level of the battery, combined with power aware network protocols, improves network lifetime, reliability, and adaptability. The amplifier is designed in $0.18 \mu \mathrm{m}$ CMOS process and operates with nominal $1.2 \mathrm{~V}$ supply.
\end{abstract}

\section{INTRODUCTION}

The integrated circuit technology, supporting the ongoing miniaturization of sensing, processing, storage, and transmission, enables new kinds of embedded systems. These systems are distributed and deployed in environments where conditions change dynamically. They comprise a collection of devices, each acting as a node of a physical communication network collecting and disseminating a wide range of environmental data.

Low data rate sensor networks are rapidly emerging as a major new player in the information technology arena. Currently many research efforts focus on the design of small, low-cost, lightweight, fully integrated, wireless communication sensor nodes. The network lifeblood, fueling range, versatility, and reliability, comes from the available power sources and from the efficiency in their usage. Each node has a battery or an energy storage device and may have means of extracting energy from the environment (solar, vibration, temperature difference) [1] or receiving wirelessly transmitted energy from a base station [2].

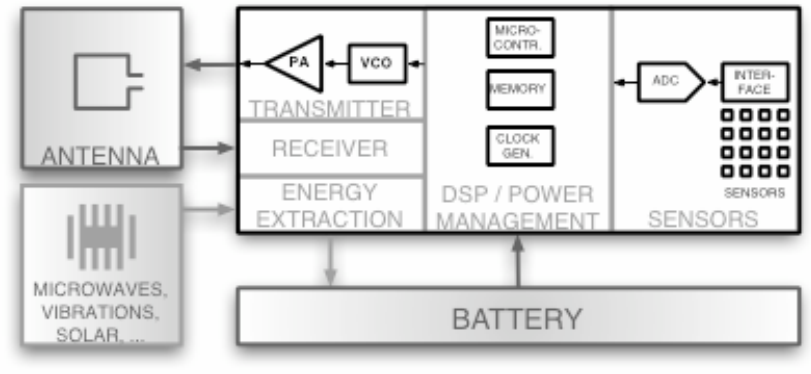

Figure 1. Block diagram of the micro-sensor.

Physical dimensions and cost of energy extraction/storage devices, limit the node power budget to a level somewhere below $100 \mu \mathrm{W}$ to have a reasonable network lifetime [2], [3]. In nodes organized as in Fig. 1, the most power-hungry block is the transmitter, which generally uses more than $50 \%$ of the available power.

Considering power dissipation, the key component in the whole sensor node is the power amplifier (PA). The challenges faced in designing a power amplifier for wireless sensor networks are quite different with respect to its counterpart of conventional wireless transmitters (e.g. Bluetooth, Wi-Fi). Here the power constraints typically limit transmission duty cycle to less than $1 \%$, so that occasional bursts of data pockets are sent with a low data rate (a few tens of $\mathrm{Kbit} / \mathrm{s}$ ) to neighboring nodes or to a base station in a few tens of meters range. Power efficiency is the most important requirement, which directly affects network range, lifetime, and reliability. In addition a power control circuit must be designed in order to change the output power for various transmission distances, variable fading conditions, and charge level of the battery. Finally, since the power amplifier must be off when idle and turned on just for short transmission bursts, the turn-on transient must be very fast to reduce the overhead power dissipation.

In this paper we present a high-efficiency class-E switched-mode power amplifier suitable for wireless

This work has been supported by the National Science Foundation grant ECS-0225528. 
networked micro-sensors systems. Switched-mode power amplifiers have a better efficiency than linear amplifiers (A, $\mathrm{B}, \mathrm{A} / \mathrm{B}$ ), thus a non-linear modulation scheme (i.e. FSK) is the best choice for low-power consumption. In addition the proposed transmitter uses the $433 \mathrm{MHz}$ ISM band, which gives a shorter path-loss than higher frequencies, allows more power-efficient transmitter circuits, and still provides enough bandwidth for the transmitted signal and allows the design of a relatively small antenna. The amplifier, together with the power control circuit, has been designed in $0.18 \mu \mathrm{m}$ CMOS process and operates with nominal $1.2 \mathrm{~V}$ supply.

The first part of the paper describes design issues and trade-offs. The following section discusses simulation results and the last section offers our concluding remarks.

\section{DESIGN CONSIDERATIONS}

\section{A. Approach}

The schematic and circuit waveforms of a class $E$ tuned amplifier are shown in Fig. 2. It consists of a transistor (M1), operated as a switch, an inductor $L_{0}$, large enough that the current through it can be considered almost constant, and a parallel load network $\left(L-C-R_{A}-C_{1}\right)$. The signal $V_{I N}$ is a square waveform generated by the preamplifier, which turns on and off M1 at the operating frequency $(433 \mathrm{MHz})$. The circuit is characterized by two resonant frequencies: when M1 is on, the series-resonant circuit consists of $C, L$, and $R_{A}$; when $\mathrm{M} 1$ is off, consists of $C_{1}, C, L$, and $R_{A}$. For optimum operation (i.e. minimum power dissipated by M1), the drain voltage $V_{D}$ and its derivative $d V_{D} / d t$ must be zero when M1 turns on (at $\omega t=2 \pi)$.

Fig. 2 shows possible current and voltage waveforms for optimum operation. This condition, for a given operating frequency, is met by setting the values of $C_{1}, C, L$, and $R_{A}$, and by choosing a proper duty cycle. A detailed analytical model for the circuit and the derivation of the steady-state waveforms are described in [4].

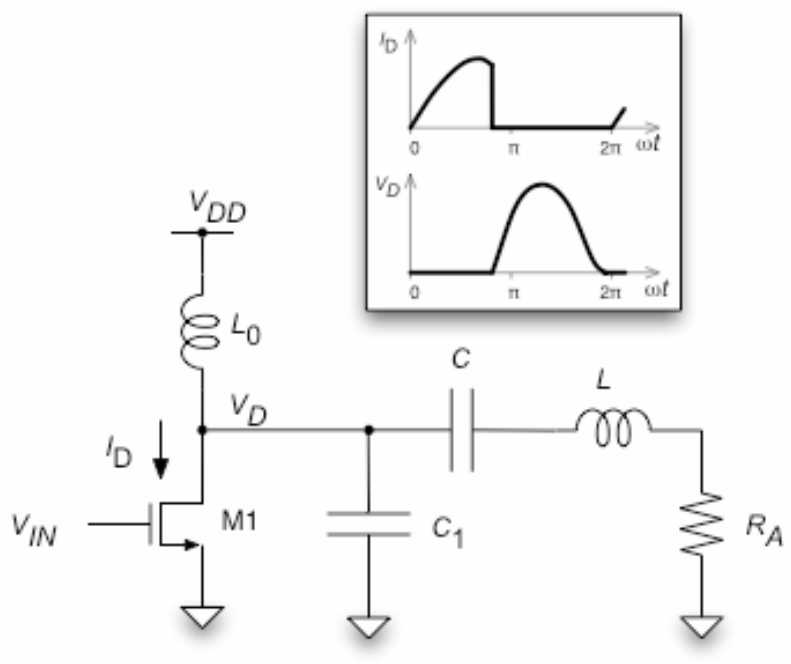

Figure 2. Power amplifier and circuit waveforms.

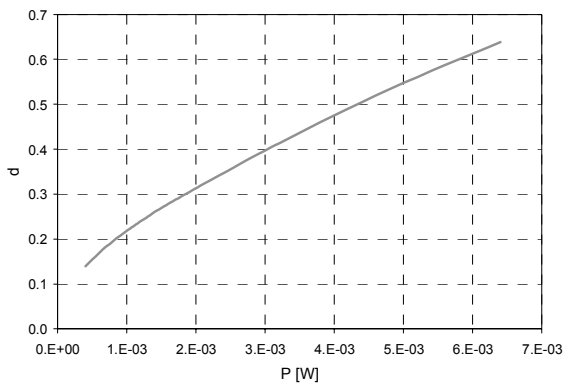

Figure 3. Duty cycle vs. output power.

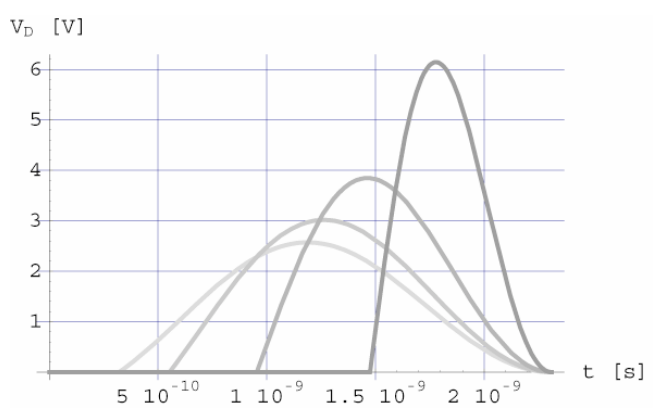

Figure 4. Voltage waveforms for variable output power levels.

The output power of the circuit is related to the waveforms, which in turn depend on $V_{D D}$, the operating frequency, the duty cycle, and the values of $C_{1}, C, L$, and $R_{A}$. The variation of any of these parameters affects the output power, but, in general, drives the working conditions away from the maximum efficiency point.

Changing the output power, while keeping the conditions for maximum efficiency, requires the simultaneous variation of more than one of the mentioned parameters. Parameters that can actually be changed in an integrated circuit are the duty cycle (that can be accomplished by the preamplifier, Sec. II.B), the capacitor $C_{1}$ (additional capacitors can be connected in parallel using $n \mathrm{MOS}$ switches), the resistor $R_{A}$ (which in practical applications results in a matching network, whose input impedance depends on the values of capacitors that can be varied the same way as $C_{1}$, Sec. II.C).

The values of the parameters corresponding to any desired value of output power can be calculated starting from the equation describing the steady-state waveform for $V_{D}$ and applying the optimum turn-on conditions $\left(V_{D}=0\right.$ and $d V_{D} /$ $d t=0)$. Figs. 3, 4, and 5 present results from numerical simulations. Fig. 3 shows the required duty cycle for maximum efficiency when the output power ranges from 0.4 to $6.4 \mathrm{~mW}$. Drain voltage waveforms corresponding to different output power levels $(0.4,1.2,3.2,6.4 \mathrm{~mW})$ are plotted in Fig. 4.

The same parameters variation can also be used to maintain a constant output power while $V_{D D}$ changes (for example because of battery discharge). Fig. 5 presents voltage waveforms for a constant output power $(1.2 \mathrm{~mW})$, and $V_{D D}=0.8,1.2$, and $1.6 \mathrm{~V}$. 


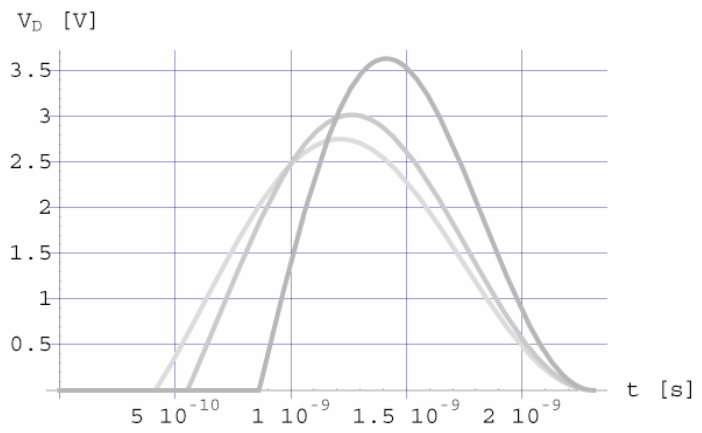

Figure 5. Voltage waveforms for constant output power and variable $V_{D D}$.

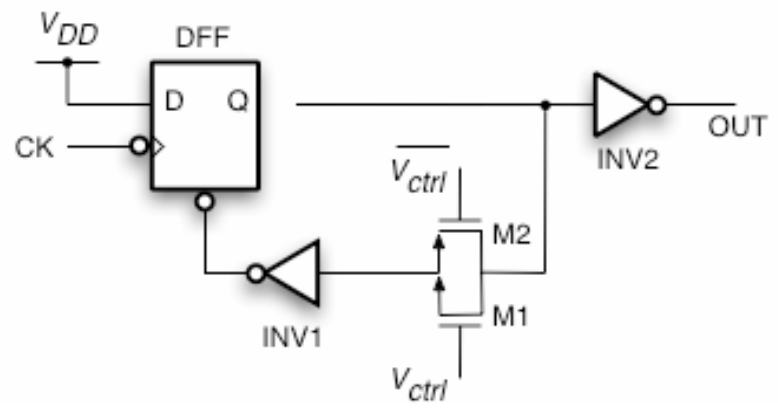

Figure 6. Duty cycle modulator (DCM).

\section{B. Preamplifier with Duty Cycle Adjustment}

The duty cycle adjustment of carrier frequency is obtained using the circuit shown in Fig. 6. The pulse is adjusted with a variable resistor created by pass transistors M1-M2 and control voltages. The circuit works as follows, whenever a rising edge occurs at the input, the output of DFF starts rising, this voltage is applied to the input of the inverter INV1 with an RC delay, determined by the on resistance of the pass transistors and the input capacitance of the inverter. Then, INV1 resets DFF. Hence the pulse width is determined by the propagation delay from DFF's Q terminal to INV1's output terminal plus DFF's reset terminal to Q terminal. Fig. 7 shows the variation of the duty cycle with respect to the applied control voltage. The transient simulation results showing the output of the duty cycle modulator is given in Fig. 8. The circuit dissipates $170 \mu \mathrm{W}$ at $433 \mathrm{MHz}$. Although, it is not shown here, digital programming of the RC delay line is also possible. For the digital programming scheme, transistors $\mathrm{M} 1$ and $\mathrm{M} 2$ are designed as multi finger pass transistors that can be turn on or off digitally so that effective channel width of the pass transistors can be programmed.

\section{Capacitively Programmable Load}

In order to change the equivalent resistive load with respect to different output power (or equivalently duty cycle setting), a capacitively programmable load is designed. The whole class E power amplifier schematic is given in Fig. 9, and capacitively programmable load consists of the components $C_{2}, C_{3}, L_{1}$, and $R_{\mathrm{A}}$. The real part of the power amplifier's load in proximity to the resonance frequency can be expressed as:

$$
R_{L}=\frac{R_{A}}{1+\left(R_{A}^{2} L_{1}^{2}-2 L_{1} C_{3}\right) \omega^{2}+L_{1}^{2} C_{3}^{2} \omega^{4}}
$$

As it is clear from (1), effective load near the resonance frequency can be modified using $C_{3}$. Since, changing the value of $C_{3}$ changes also equivalent resonance frequency of the load, the value of another capacitor, i.e. $C_{2}$, is changed in correlation with the changes of $C_{3}$ so that overall resonance frequency can be set at the desired $433 \mathrm{MHz}$.

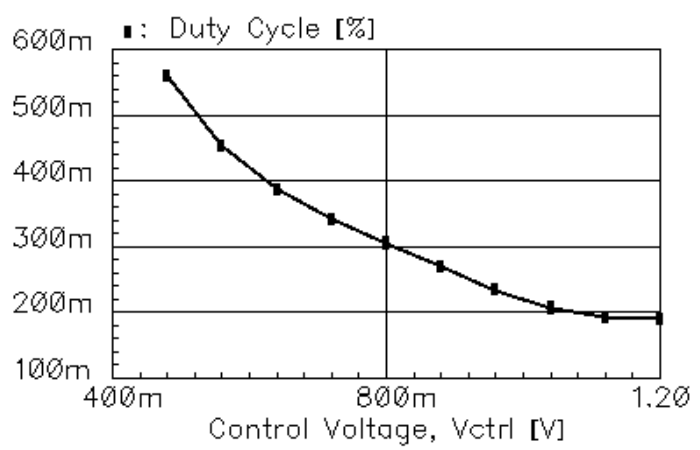

Figure 7. The variation of the duty cycle with control voltage.

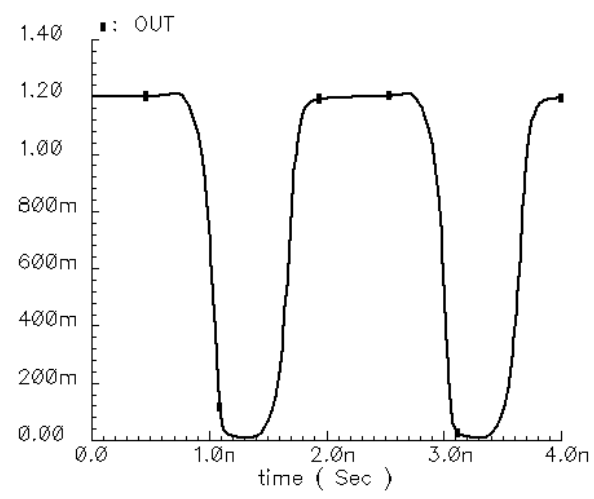

Figure 8. Transient simulation result of the duty cycle modulator.

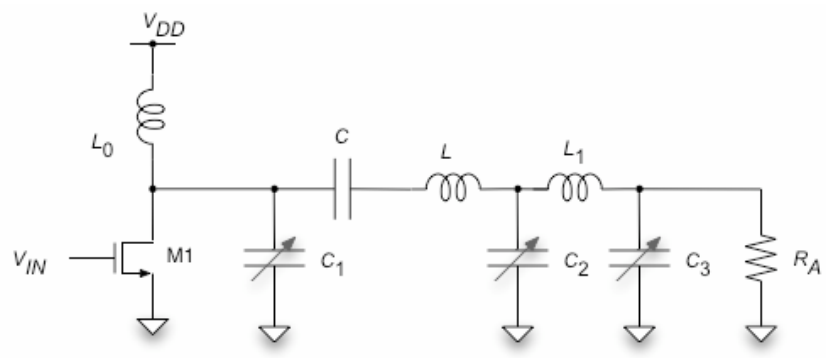

Figure 9. Circuit schematic of the power amplifier. 


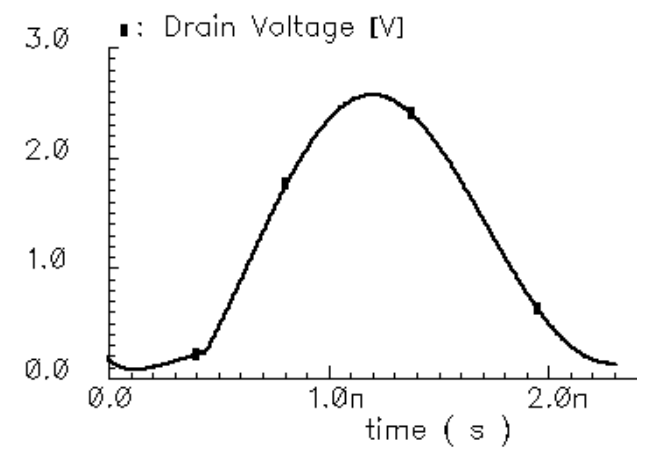

Figure 10. Drain voltage of switch transistor M1 (PSS analysis).

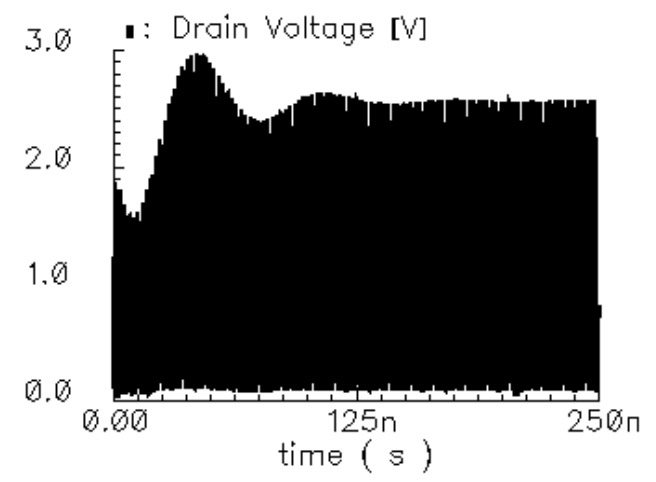

Figure 11. Drain voltage of switch transistor M1 (transient analysis).

\section{RESULTS}

To prove the validity of the proposed scheme, the class-E power amplifier shown in Fig. 9 has been extensively simulated. Table 1 shows the obtained output power and power added efficiency of the class-E power amplifier for different $C_{1}, C_{2}, C_{3}$ and duty cycle values. Fig. 10 shows the drain voltage of the switch transistor M1 for the output power level of $-0.211 \mathrm{dBm}$ obtained using periodic steady state analysis (PSS). Fig. 11 shows the initial transient at the switch drain terminal. The power added efficiency of the class-E amplifier, together the PAE of the whole power amplifier including the duty cycle modulator with respect to the output power is shown in Fig. 12. Class-E power amplifier shows more than $87 \%$ throughout the whole output power range and of course overall PAE decrease with the decrease of the output power because of the constant power consumption of the duty cycle modulator (DCM).

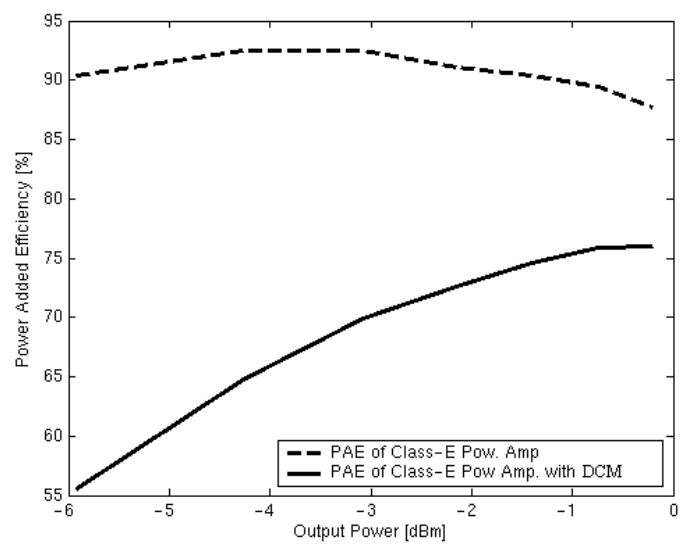

Figure 12. Power Added Efficiency (PAE) of the class-E power amplifier: a) alone; b) with preamplifier, i.e. duty cycle modulator.

\section{CONCLUSION}

Wireless sensor networks set new challenges in designing low-power communication circuits. The power available to each micro-sensor node is a vital resource for the functioning a sensor network and the transmitted power is a substantial part of it. This paper presents a high-efficiency power amplifier, which is particularly well suited for wireless networked micro-sensors systems. Simulation results shows that it keeps optimum efficiency conditions, with PAE over $87 \%$, while delivering variable output power, from -4.262 to $-0.211 \mathrm{dBm}$, without needing for additional power control mechanisms.

\section{REFERENCES}

[1] J.M. Rabaey, J. Ammer, T. Karalar, S. Li, B. Otis, M. Sheets, T. Tuan, "Picoradios for wireless sensor networks: the next challenge in ultra-low-power design," Digest of Technical Papers of the 2002 Solid-State Circuit Conference, Vol. 1, pp. 201-202.

[2] S. Gregori, Y. Li, H. Li, J. Liu and F. Maloberti, "2.45 GHz power and data transmission for a low-power autonomous sensor platform," Proceedings of the 2004 International Symposium on Low Power Electronics and Design, pp. 269-273, Aug. 2004.

[3] Y.-H. Chee, J. Rabaey, A.M. Niknejad, "A class A/B low power amplifier for wireless sensor networks," Proceedings of the 2004 International Symposium on Circuits and Systems, Vol. 4, pp. 409412, May 2004.

[4] M. Kazimierczuk and K. Puczko, "Exact analysis of class E tuned power amplifier at any Q and switch duty cycle," IEEE Trans. on Circuits and Systems, Vol. 34, Issue 2, pp. 149-159, Feb. 1987.

TABLE I. OUtPut POWER AND POWER ADDED EFFICIENCY FOR DIFFERENT COMPONENT VALUES AND Duty CYCLE

\begin{tabular}{|c|c|c|c|c|c|c|c|c|}
\hline Duty Cycle & $\boldsymbol{L}[\mathbf{H}]$ & $\boldsymbol{C}[\mathbf{F}]$ & $\boldsymbol{C}_{\mathbf{1}}[\mathbf{F}]$ & $\boldsymbol{L}_{\mathbf{1}}[\mathbf{H}]$ & $\boldsymbol{C}_{\mathbf{2}}[\mathbf{F}]$ & $\boldsymbol{C}_{\mathbf{3}}[\mathbf{F}]$ & Output Power $[\mathbf{d B m}]$ & Power Added Efficiency $[\%]$ \\
\hline 0.178 & $1.00 \mathrm{E}-07$ & $6.50 \mathrm{E}-11$ & $1.25 \mathrm{E}-12$ & $9.19 \mathrm{E}-09$ & $2.16 \mathrm{E}-11$ & $2.48 \mathrm{E}-11$ & -4.262 & 92.46 \\
\hline 0.205 & $1.00 \mathrm{E}-07$ & $6.50 \mathrm{E}-11$ & $1.20 \mathrm{E}-12$ & $9.19 \mathrm{E}-09$ & $2.20 \mathrm{E}-11$ & $2.81 \mathrm{E}-11$ & -3.075 & 92.44 \\
\hline 0.230 & $1.00 \mathrm{E}-07$ & $6.50 \mathrm{E}-11$ & $1.15 \mathrm{E}-12$ & $9.19 \mathrm{E}-09$ & $2.20 \mathrm{E}-11$ & $3.05 \mathrm{E}-11$ & -2.153 & 91.10 \\
\hline 0.253 & $1.00 \mathrm{E}-07$ & $6.50 \mathrm{E}-11$ & $1.10 \mathrm{E}-12$ & $9.19 \mathrm{E}-09$ & $2.19 \mathrm{E}-11$ & $3.24 \mathrm{E}-11$ & -1.407 & 90.40 \\
\hline 0.275 & $1.00 \mathrm{E}-07$ & $6.50 \mathrm{E}-11$ & $1.05 \mathrm{E}-12$ & $9.19 \mathrm{E}-09$ & $2.18 \mathrm{E}-11$ & $3.41 \mathrm{E}-11$ & -0.741 & 89.42 \\
\hline 0.296 & $1.00 \mathrm{E}-07$ & $6.50 \mathrm{E}-11$ & $1.00 \mathrm{E}-12$ & $9.19 \mathrm{E}-09$ & $2.16 \mathrm{E}-11$ & $3.56 \mathrm{E}-11$ & -0.211 & 87.94 \\
\hline
\end{tabular}

